\title{
emm type diversity of $\beta$-haemolytic streptococci recovered in Chennai, India
}

Beta-haemolytic streptococci (BHS) are widely spread human pathogens, and include group A, G and C streptococci. Group A streptococci (GAS) are mostly found in the throat and on the skin and commonly cause pharyngitis and impetigo, although they are most notorious for causing a broad spectrum of severe invasive disease (Cunningham, 2000). Recent studies have indicated that group C and G streptococci (GCS, GGS) also carry Streptococcus pyogenes superantigen genes and have been reported with increasing frequency from a variety of severe infections (Davies et al., 2005; Kalia \& Bessen, 2003). The $M$ type specific typing scheme for GAS, based upon the hypervariable region of the $\mathrm{emm}$ gene, also applies to GCS and GGS (Bisno et al., 1996; Collins et al., 1992).

The objective of this study was to investigate the diversity of BHS in Chennai, south India, by emm gene sequencing.

Ninety-five isolates comprising 49 GGS, 34 GAS and 12 GCS from our laboratory collection were emm typed/subtyped and T typed according to standard protocols (http://www.cdc.gov/ncidod/biotech/strep/ protocol_emm-type.htm). Two isolates were from blood; the remainder were from non-invasive infections. With the exception of eight isolates, all were recovered from individuals within the age range of 2-15 years.

Approval for the research was obtained from the Ethical Committee of the institution.

Forty-nine of the 95 isolates $(51.6 \%)$ were found to be GGS, 12 (12.6\%) were GCS and $34(35.8 \%)$ were grouped as GAS. All 61 GGS/GCS and one GAS isolate (emm49.4) were bacitracin-resistant.

Fourteen emm types were identified among the 49 GGS isolates, the most common type being stG485 (12/49) followed by stGLP1 (6/49) (Table 1). stG485 was also the most common type reported from group $\mathrm{G}$ bacteraemia in Jerusalem, from toxigenic GGS strains in Japan and from GCS strains in Portugal, and was found from a GAS strain in Hungary (CohenPoradosu et al., 2004; Hashikawa et al., 2004; Pinho et al., 2006; Krucsó et al., 2005). stG6792 and stGLP1 each

represented $25 \%$ of the pyoderma isolates, and have also been reported from Portugal (Pinho et al., 2006). Four of our isolates were $s t C 5345$, a type originally reported from invasive isolates in Argentina (Lopardo et al., 2005); other GGS emm types in our study included stC36, stG2078, stC1400, stG4831, stG643, stG10 and stG2574, which have been previously reported from invasive infections (Horii et al., 2006; Cohen-Poradosu et al., 2004; Pinho et al., 2006; Lopardo et al., 2005).

The reported prevalence of GCS/GGS carriage and disease incidence varies greatly worldwide. Most studies originate from temporal climate regions of the northern hemisphere and limited data are available from tropical regions (McDonald et al., 2007).

Twelve GCS were isolated, representing five different emm types; the most prevalent were $s t G 245.0(4 / 12,33.33 \%)$ and $s t C 1400(4 / 12,33.33 \%)$. Subtype stC1400.0 was found in both GGS and GCS (Table 1). One type found among the GCS, stCK401, was originally found in a GAS isolate recovered in the Northern Territory of Australia (see http://www.cdc.gov/ ncidod/biotech/strep/types_emm103-124. htm).

We found two new emm sequence types among the GGS and GCS in this study, stG866, found in two GGS isolates associated with pharyngotonsillitis, and stC922.0, from a GCS isolate associated with pharyngitis.

Thirty-four GAS isolates could be typed as 22 distinct emm types, reflecting high strain diversity $(22 / 34,64.7 \%)$. The most common emm type was emm49, accounting for four isolates and two different subtypes, followed by emm 82 and emm74. We found two non-invasive isolates each of emm121, emm118, emm71 and emm105. Two independent type emm109 invasive isolates were also identified. Other emm types were represented by only one isolate each. We had a few types rarely or never reported in the USA, which included emm8, emm55, emm95, emm71, emm74, emm104, emm105, emm106, emm109 and st2460 (Shulman et al., 2004). In contrast, six of these types (emm55, emm71, emm74, emm104, emm105 and emm106) were observed among a recent collection of non-invasive isolates recovered in western Nepal (Sakota et al., 2006). Five of these types (emm8, emm74, emm95, emm106 and emm109) were found during a carriage study in Ethiopia (Abdissa et al., 2006), and four of these types (emm55, emm71, emm74 and emm95) were found during invasive GAS surveillance in Israel (Moses et al., 2003). We also found, similar to previous observations among isolates recovered in Nepal, that $M$ types did not have the characteristic $\mathrm{T}$ type associations observed in the USA and western Europe (Johnson et al., 2006). These unusual associations (for the USA and western Europe) included T12/27/emm49, TB3264/ emm82, T14/27/Imp19/emm28, T2/emm75 and T8/emm87. The T12/emm49, TB3264/ emm82 and $\mathrm{T} 2 / e m m 75$ associations were also observed among the isolates recovered in Nepal, and further genetic characterization revealed the Nepalese isolates to be of completely different clonal lineages to the corresponding emm 28 , emm49, emm82, emm75 and emm87 isolates recovered in the USA (Sakota et al., 2006).

The prevalence of emm-typable GGS/GCS in this region could reflect a high association of these strains with noninvasive infections, and could also conceivably reflect significant association 
Table 1. emm subtypes and groups of BHS recovered in Chennai

\begin{tabular}{|c|c|}
\hline emm subtype (T type if group A) & No. of isolates \\
\hline \multicolumn{2}{|l|}{ GAS } \\
\hline emm49.0 (T14, T27/12) & 2 \\
\hline emm49.4 (T27/12, T12) & 2 \\
\hline emm74.0 (T3/13/B3264/5/27/44; T3/13/B3264; B3264) & 3 \\
\hline emm82.1 (T14; T3/13/B3264) & 2 \\
\hline emm82.0 (ТB3264) & 1 \\
\hline emm71.0 (T1/3/13/B3264; 13/B3264) & 2 \\
\hline emm118.0 (T27/12; T-nontypeable) & 2 \\
\hline emm109.0 (T6) & 1 \\
\hline emm109.1 (T6) & 1 \\
\hline emm121.0 (T-nontypeable $\{2\}$ ) & 2 \\
\hline emm105.0 (TB3264; T-nontypable) & 2 \\
\hline st2460.1 (T3/13/B3264) & 1 \\
\hline emm8.0 (T-nontypeable) & 1 \\
\hline emm28.5 (T27/14/Imp19) & 1 \\
\hline emm55.0 (T-nontypeable) & 1 \\
\hline emm58.8 (T-nontypeable) & 1 \\
\hline emm66.0 (T4) & 1 \\
\hline emm75.1 (T2) & 1 \\
\hline emm77.0 (T-nontypeable) & 1 \\
\hline emm87.0 (T8) & 1 \\
\hline emm93.0 (T8) & 1 \\
\hline emm95.0 (T3/13/B3264) & 1 \\
\hline emm103.0 (T2/4) & 1 \\
\hline emm104.0 (T-nontypeable) & 1 \\
\hline emm106.0 (T5/27/44) & 1 \\
\hline \multicolumn{2}{|l|}{ GCS } \\
\hline stC1400.2 & 3 \\
\hline stC1400.0 & 1 \\
\hline stG245.0 & 4 \\
\hline stCK401.2 & 1 \\
\hline stC922.0 & 1 \\
\hline st $G 653.0$ & 2 \\
\hline \multicolumn{2}{|l|}{ GGS } \\
\hline st $G 485.0$ & 12 \\
\hline stC1400.0 & 3 \\
\hline stGLP1.0 & 6 \\
\hline stC5345.0 & 4 \\
\hline stC5345.2 & 1 \\
\hline st $G 6792.0$ & 4 \\
\hline stG6792.3 & 1 \\
\hline stC36.0 & 3 \\
\hline st G2078.0 & 2 \\
\hline st $G 2078.2$ & 1 \\
\hline stC74a.0 & 2 \\
\hline st $G 4831.0$ & 2 \\
\hline st $G 643.0$ & 1 \\
\hline stG643.1 & 1 \\
\hline stKNB7.0 & 2 \\
\hline stG866.0 & 2 \\
\hline st $G 2574.0$ & 1 \\
\hline st $G 10.0$ & 1 \\
\hline
\end{tabular}

with invasive disease. As with other limited studies of non-invasive isolates recovered in tropical or subtropical climates (Abdissa et al., 2006; Sakota et al., 2006), we found a high degree of GAS emm type diversity, indicating a potential challenge for a multivalent $M$ type specific vaccine strategy and further need for surveillance of GAS isolates from disease cases in these regions.

Thangam Menon, ${ }^{1}$ Charmaine Lloyd, ${ }^{1}$ Balaraman Malathy, ${ }^{1}$ Varja Sakota, ${ }^{2}$ Delois Jackson ${ }^{2}$ and Bernard Beall ${ }^{2}$

${ }^{1}$ Department of Microbiology, Dr A. L. Mudaliar Post Graduate Institute of Basic Medical Sciences, Taramani, University of Madras, Chennai, India

${ }^{2}$ Respiratory Diseases Branch, Centers for Disease Control and Prevention, Atlanta, GA, USA

Correspondence: Thangam Menon (thangam56@gmail.com)

Abdissa, A., Asrat, D., Kronvall, G., Shittu, B., Achiko, D., Zeidan, M., Yamuah, L. K. \& Aseffa, A. (2006). High diversity of group A streptococcal emm types among healthy school children in Ethiopia. Clin Infect Dis 42, 1362-1367.

Bisno, A. L., Collins, C. M. \& Turner, J. C. (1996). $\mathrm{M}$ proteins of group $\mathrm{C}$ streptococci isolated from patients with acute pharyngitis. J Clin Microbiol 34, 2511-2515.

Cohen-Poradosu, R., Jaffe, J., Lavi, D., Grisariu-Greenzaid, S., Nir-Paz, R., Valinsky, L., Dan-Goor, M., Block, C., Beall, B. \& Moses, A. E. (2004). Group G streptococcal bacteremia in Jerusalem. Emerg Infect Dis 10, 1455-1460.

Collins, C. M., Kimura, A. \& Bisno, A. L. (1992). Group G streptococcal M protein exhibits structural features analogous to those of class I $\mathrm{M}$ protein of group A streptococci. Infect Immun 60, 3689-3696.

Cunningham, M. W. (2000). Pathogenesis of group A streptococcal infections. Clin Microbiol Rev 13, 470-511.

Davies, M. R., Tran, T. N., McMillan, D. J., Gardiner, D. L., Currie, B. J. \& Sriprakash, K. S. (2005). Inter-species genetic movement may blur the epidemiology of streptococcal diseases in endemic regions. Microbes Infect 7 , 1128-1138.

Hashikawa, S., linuma, Y., Furushita, M., Ohkura, T., Nada, T., Tori, K., Hasegawa, T. \& Ohta, M. (2004). Characterization of group C and $\mathrm{G}$ streptococcal strains that cause streptococcal toxic shock syndrome. J Clin Microbiol 42, 186-192. 
Horii, T., Izumida, S., Takeuchi, K., Tada, T., Ishikawa, J. \& Tsuboi, K. (2006). Acute peritonitis and salpingitis associated with streptococcal toxic shock syndrome caused by Lancefield group G $\alpha$-haemolytic Streptococcus dysgalactiae subsp. equisimilis. J Med Microbiol 55, 953-956.

Johnson, D. R., Kaplan, E. L., VanGheem, A., Facklam, R. R. \& Beall, B. (2006).

Characterization of group A streptococci (Streptococcus pyogenes): correlation of Mprotein and $\mathrm{emm}$-gene type with T-protein agglutination pattern and serum opacity factor. J Med Microbiol 55, 157-164.

Kalia, A. \& Bessen, D. E. (2003). Presence of streptococcal pyrogenic exotoxin $\mathrm{A}$ and $\mathrm{C}$ genes in human isolates of group $\mathrm{G}$ streptococci. FEMS Microbiol Lett 219, 291-295.

Krucsó, B., Csiszár, K., Gacs, M. \& Pászti, J. (2005). Molecular typing of Hungarian Streptococcus pyogenes isolates. In Abstracts of the 15th European Congress of Clinical Microbiology and Infectious Diseases Copenhagen, Denmark, abstract 1134-02-364. European Society of Clinical Microbiology and Infectious

Diseases.

Lopardo, H. A., Vidal, P., Sparo, M., Jeric, P., Centron, D., Facklam, R. R., Paganini, H., Pagniez, N. G., Lovgren, M. \& Beall, B. (2005). Six-month multicenter study on invasive infections due to Streptococcus pyogenes and Streptococcus dysgalactiae subsp. equisimilis in Argentina. J Clin Microbiol 43, 802-807.

McDonald, M., Towers, R. J., Andrews, R. M., Carapetis, J. R. \& Currie, B. J. (2007).

Epidemiology of Streptococcus dysgalactiae subsp. equisimilis in tropical communities, Northern Australia. Emerg Infect Dis 13, 1694-1700.

Moses, A. E., Hidalgo-Grass, C., Dan-Goor, M., Jaffe, J., Sheitzigovsky, I., Ravins, M., Korenman, Z., Cohen-Poradosu, R. \& Nir-Paz,
R. (2003). emm typing of M nontypable invasive group A streptococcal isolates in Israel. J Clin Microbiol 41, 4655-4659.

Pinho, M. D., Melo-Cristino, J. \& Ramirez, M. (2006). Clonal relationships between invasive and noninvasive Lancefield group C and G streptococci and emm-specific differences in invasiveness. J Clin Microbiol 44, 841-846.

Sakota, V., Fry, A. M., Lietman, T. M., Facklam, R. R., Li, Z. \& Beall, B. (2006). Genetically diverse group A streptococci from children in far-western Nepal share high genetic relatedness with isolates from other countries. J Clin Microbiol 44, 2160-2166.

Shulman, S. T., Tanz, R. R., Kabat, W., Kabat, K., Cederlund, E., Patel, D., Li, Z., Sakota, V., Dale, J. B., Beall, B. \& the US Streptococcal Pharyngitis Surveillance Group (2004). Group A streptococcal pharyngitis serotype surveillance in North America, 2000-2002. Clin Infect Dis 39, 325-332. 